\title{
Nucleation of vortices by rapid thermal quench
}

\author{
I.S. Aranson ${ }^{1}$, N.B. Kopnin ${ }^{1,2}$ and V.M. Vinokur ${ }^{1}$ \\ ${ }^{1}$ Argonne National Laboratory, 9700 South Cass Avenue, Argonne, IL 60439 \\ ${ }^{2}$ L.D. Landau Institute for Theoretical Physics, 117334 Moscow, Russia
}

(May 12, 2018)

\begin{abstract}
We show that vortex nucleation in superfluid ${ }^{3} \mathrm{He}$ by rapid thermal quench in the presence of superflow is dominated by a transverse instability of the moving normal-superfluid interface. Exact expressions for the instability threshold as a function of supercurrent density and the front velocity are found. The results are verified by numerical solution of the Ginzburg-Landau equation.
\end{abstract}

PACS: 67.57.Fg,74.40.+k,05.70.Fh

Formation of topological defects under a rapid quench is a fundamental problem of contemporary physics promising to shed a new light on the early stages of the evolution of the Universe. For homogeneous cooling a fluctuation-dominated formation mechanism has been suggested by Kibble and Zurek (KZ) [1] 3]. Normally, cooling is associated with an inhomogeneous temperature distribution accompanied by a phase separating interface which moves through the system as temperature decreases. A generalization of the $\mathrm{KZ}$ scenario was suggested in Ref. [4] for inhomogeneous phase transitions in superfluids: if the front moves faster than the normal-superfluid interface a large supercooled region which is left behind becomes unstable towards fluctuation-induced nuclei.

Superfluid ${ }^{3} \mathrm{He}$ offers a unique "testing ground" for rapid phase transitions [5]. Recent experiments where a rotating superfluid ${ }^{3} \mathrm{He}$ was locally heated well above the critical temperature by absorption of neutrons [6] revealed vortex formation under a rapid second-order phase transition. The TDGL analysis was applied to study a propagating normal-superfluid interface under inhomogeneous cooling [7] and the formation of a large supercooled region was confirmed. The fluctuationdominated mechanism may thus be responsible for creation of initial vortex loops. It is commonly accepted that these initial vortex loops are further inflated by the superflow and give rise to a macroscopic number of large vortex lines filling the bulk superfluid.

In this Letter we report a novel mechanism of vortex formation which overtakes growth of the initial loops appearing in the supercooled region. We study the entire process of vortex formation in the presence of a superflow using TDGL dynamics. We take into account the temperature evolution due to thermal diffusion. We find analytically and confirm by numerical simulations that the normal-superfluid interface becomes unstable with respect to transverse undulations in the presence of a superflow. These undulations quickly transform into large primary vortex loops which then separate themselves from the interface. Simultaneously, a very large number of small secondary vortex/antivortex nuclei are cre- ated in the supercooled region by fluctuations resembling the conventional KZ mechanism. The primary vortex loops screen out the superflow in the inner region causing the annihilation of the secondary vortex/antivortex nuclei. The number of surviving vortex loops is thus much smaller than what anticipated from the KZ conjecture.

Model. - We use the TDGL model for a scalar order parameter $\psi$ ignoring the non-relevant complexity of the ${ }^{3} \mathrm{He}-$ specific multicomponent order parameter:

$$
\partial_{t} \psi=\Delta \psi+(1-f(\mathbf{r}, t)) \psi-|\psi|^{2} \psi+\zeta(\mathbf{r}, t) .
$$

Here $\Delta$ is the three-dimensional (3D) Laplace operator. Distances and time are measured in units of the coherence length $\xi\left(T_{\infty}\right)$ and the characteristic time $\tau_{G L}\left(T_{\infty}\right)$, respectively. These quantities are taken at temperature $T_{\infty}$ far from the heated bubble. The local temperature is controlled by heat diffusion and evolves as $f(\mathbf{r}, t)=$ $E_{0} \exp \left(-r^{2} / \sigma t\right) t^{-3 / 2}$ where $\sigma$ is the normalized diffusion coefficient. $E_{0}$ determines the initial temperature of the hot bubble $T^{*}$ and is proportional to the deposited energy $\mathcal{E}_{0}$ such that $E_{0}=\mathcal{E}_{0} /\left[C\left(T_{c}-T_{\infty}\right) \xi^{3}\left(T_{\infty}\right)(\pi \sigma)^{3 / 2}\right]$ where $C$ is the heat capacity. Since the deposited energy is large compared to the characteristic superfluid energy, we assume $E_{0} \gg 1$. The time at which the temperature in the center of the hot bubble drops down to $T_{c}$ is $t_{\max }=E_{0}^{2 / 3}$. The Langevin force $\zeta$ with the correlator $\left\langle\zeta \zeta^{\prime}\right\rangle=2 T_{f} \delta\left(\mathbf{r}-\mathbf{r}^{\prime}\right) \delta\left(t-t^{\prime}\right)$ describes thermal fluctuations with a strength $T_{f}$ at $T_{c}$.

The typical values of the Ginzburg-Landau parameters for Fermi liquids are: $\tau_{G L}\left(T_{\infty}\right)=\tau_{0} /\left(1-T_{\infty} / T_{c}\right)$, $\xi\left(T_{\infty}\right) \sim \xi_{0} /\left(1-T_{\infty} / T_{c}\right)^{1 / 2}, \quad \xi_{0}=\hbar v_{F} / 2 \pi T_{c}$ and $\tau_{0}=\pi \hbar / 8 T_{c}$. The diffusion constant $\sigma \sim \ell / \xi_{0}, \ell$ is the mean free path of a quasiparticle. In ${ }^{3} \mathrm{He}, \sigma$ is very large because $\ell / \xi_{0} \sim 10^{3}$. The noise strength is $T_{f} \sim \mathrm{Gi}^{-1}\left[1-\left(T / T_{c}\right)\right]^{-1 / 2}, \mathrm{Gi}=\nu(0) \xi_{0}^{3} T_{c} \sim 10^{4}$ is the Ginzburg number and $\nu(0)$ is normal density of states.

Results. - We solved Eq. (1) by the implicit CrankNicholson method. The integration domain was equal to $150^{3}$ units of Eq. (11) with $200^{3}$ mesh points. The boundary conditions were taken as $\partial_{z} \psi=i k \psi$ with a constant $k$ at the top and the bottom of the integration domain. This implies a superflow $j_{s}=k|\psi|^{2}$ along the 
$z$-axis far away from the temperature bubble. The simulations were carried out on a massive parallel computer, the Origin 2000, at Argonne National Laboratory.

Selected results are shown in Fig. 11. One sees (Fig. [1a-c) that without fluctuations (numerical noise only [8]) the vortex rings nucleate upon the passage of the thermal front. Not all of the rings survive: the small ones collapse and only the big ones grow. Although the vortex lines are centered around the point of the quench, they exhibit a certain degree of entanglement. After a long transient period, most of the vortex rings reconnect and form the almost axisymmetric configuration.

We find that the fluctuations have a strong effect at early stages: the vortices nucleate not only at the normalsuperfluid interface, but also in the bulk of the supercooled region (Fig. $1 \mathrm{~d}$-e). However, later on, small vortex rings in the interior collapse and only larger rings (primary vortices) survive and expand (Fig. 1ff).
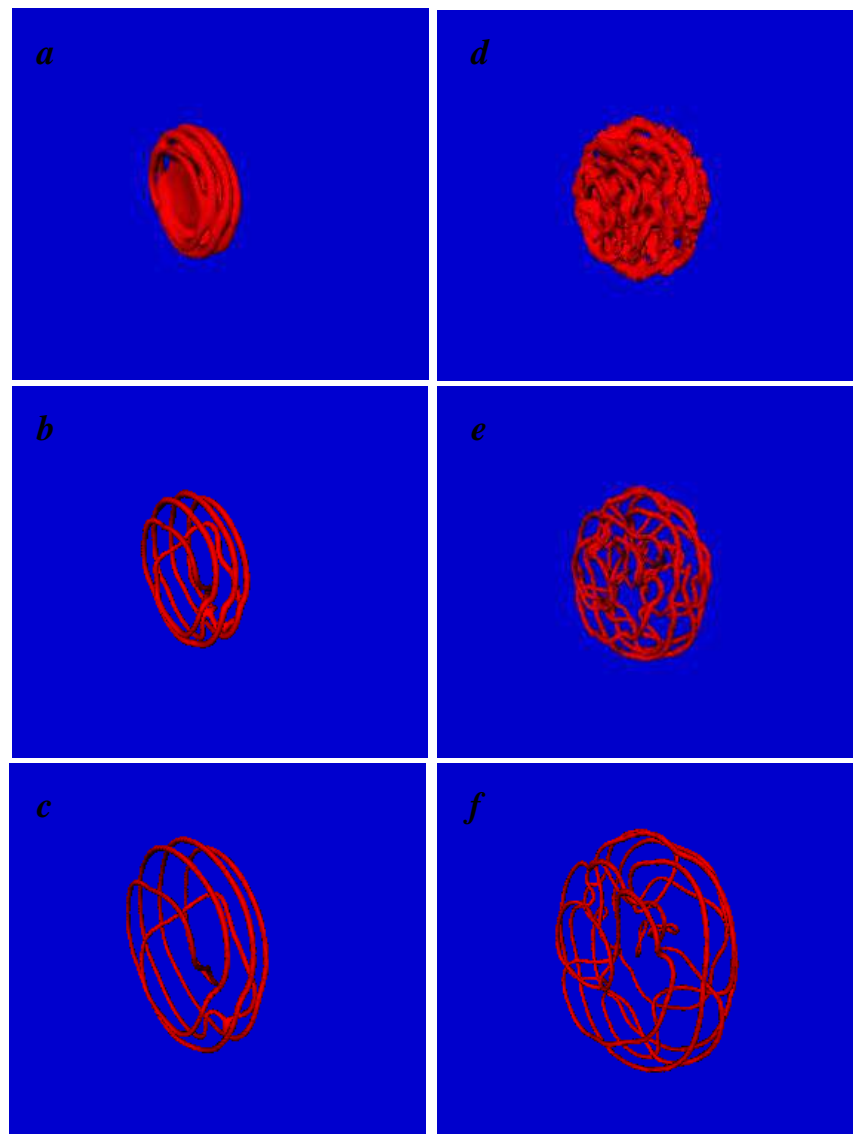

FIG. 1. 3D isosurface of $|\psi|=0.4$ for $\sigma=400, E_{0}=30$ and $k=0.5$. (a-c) $T_{f}=0$. Images are taken at times $t=36,48,80$. (d-f), $T_{f}=0.002, t=24,48,80$.

To elucidate the details of nucleation we considered an axi-symmetric version of Eq. (11) (depending on only $r$ and $z$ coordinates, $\left.\Delta=\partial_{r}^{2}+1 / r \partial_{r}+\partial_{z}^{2}\right)$ for realistic ${ }^{3} \mathrm{He}$ parameters [6]: $k \sim 1, E_{0} \gg 1$, and $\sigma \sim 10^{3}$. The domain was $500^{2}$ with $1000^{2}$ mesh points. We have found that without thermal fluctuations the vortices nucleate at the front of the normal-superfluid interface (black/white border in Fig. 2a-c) analogous to the 3D case. The initial instability is seen as a corrugation of the interface. The interface propagates towards the center, leaving the vortices behind. As thermal fluctuations are turned on, the vortex rings also nucleate in the bulk of the supercooled region (Fig. 2d) resulting in the creation of the secondary vortex/antivortex pairs. We have found that the "primary" vortices prevent the supercurrent from penetrating into the region filled with the secondary vortices. One sees that the primary vortices encircle the brighter spot in Fig. 2 indicating a larger value of the order parameter and thus a smaller value of the supercurrent. As a result the secondary vortices either annihilate with antivortices due to their mutual attraction or collapse due to the absence of the inflating superflow. Fig. 3 shows the number of vortices $N^{+}$and antivortices $N^{-}$vs time with and without fluctuations. Fluctuations initially create a very large number $\sim 10^{4}$ of vortices and antivortices in the bulk which then annihilate. The resulting amount of surviving vortices $N=N^{+}-N^{-}$is only weakly dependent on fluctuations.
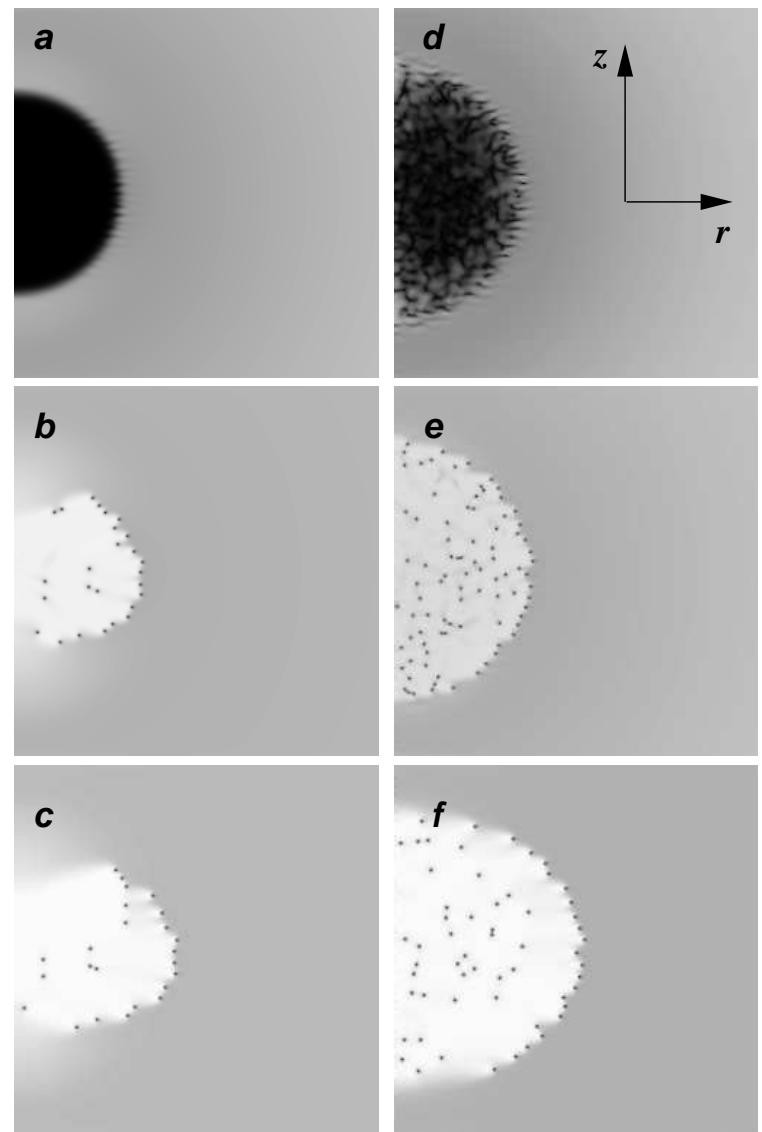

FIG. 2. Grey-coded images of $|\psi|$ for axi-symmetric Eq. (1) for $\sigma=5000, E_{0}=50$ and $k=0.5$, black corresponds to $|\psi|=0$ and white to $|\psi|=1$. Current is along the $z$-axis. Vortices are seen as black dots. (a-c) $T_{f}=0$, images are shown for $t=40,100,200$; (d-f) $T_{f}=0.002$, for $t=30,50,200$ 
Shown in Fig. 1 is the number of vortex rings $N$ vs quench parameters and applied current $k$. At small $k N$ shows threshold behavior while becoming almost linear for larger $k$ values. The deviations from linear a law appear close to the value of the critical current $k_{c}=1 / \sqrt{3}$ for a homogeneous system.

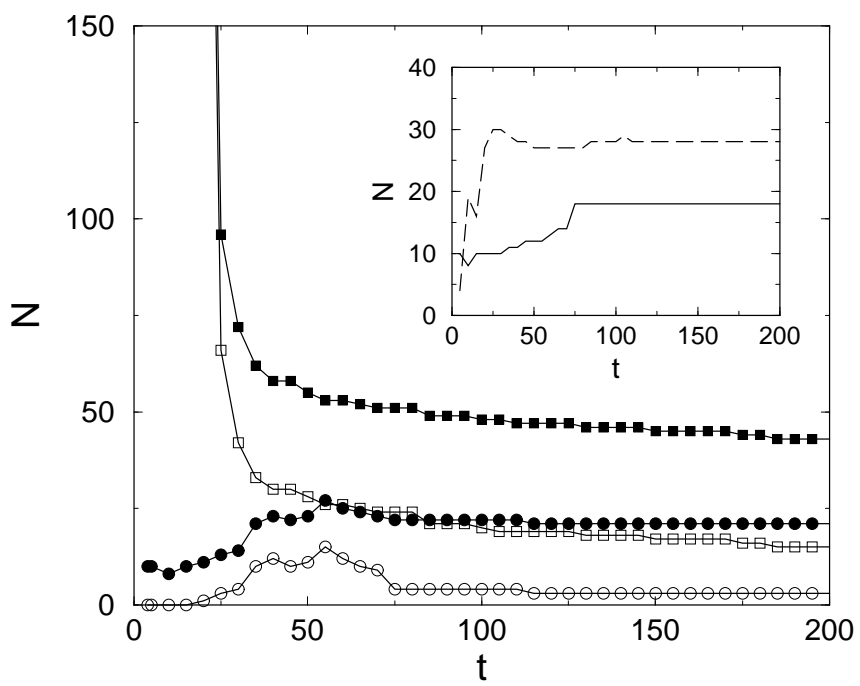

FIG. 3. Number of vortices $N^{+}$(filled symbols) and anti-vortices $N^{-}$(open symbols) vs time for $\sigma=5000, E_{0}=50$ and $k=0.5$. Circles correspond to $T_{f}=0$ and squares to $T_{f}=0.002$. Inset: $N=N^{+}-N^{-}$for $T_{f}=0$ (solid line) and $T_{f}=0.002$ (dashed line)

Stability of normal-superfluid interface.- Following Ref. [3,7], we expand the local temperature $1-f$ near $T_{c}$. Let us put $x=r_{c}-r$ where $r_{c}$ is the radius of the surface at which $T=T_{c}$ or $f=1$, i.e., $r_{c}^{2}=(3 / 2) \sigma t \log \left(t_{\max } / t\right)$. A positive $x$ is directed towards the hot region. We write $1-f(r, t) \approx-\alpha(x-v t)$ where $\alpha=-[d f / d r]_{f=1}=2 r_{c} / \sigma t$ is the local temperature gradient and $v=\left(\alpha \tau_{Q}\right)^{-1}$ is the front velocity defined through the quench rate $\tau_{Q}^{-1}=[\partial f / \partial t]_{f=1}$. We have $v=\left(3 \sigma t-2 r_{c}^{2}\right) / 4 r_{c} t$. The front starts to move towards the center at $t>t_{*}=t_{\max } / e$ and disappears at $t=t_{\max }$ when the temperature drops below $T_{c}$. The front velocity accelerates as the hot bubble collapses. Since the front radius $r_{c}$ is large compared to the coherence length, it can be considered flat. We chose the coordinate $y$ parallel to the front.

We transform to the frame moving with the velocity $v$ and perform the scaling of variables $\tilde{x}, \tilde{y}=(x, y) v, \tilde{t}=$ $t v^{2}, \tilde{\psi}=\psi / v$, and $u=v^{3} / \alpha$. The parameter $u \sim$ $\left(\sigma^{2} / t_{\max }\right) / \log ^{2}\left(t_{\max } / t\right)$ is of the order 1 in the experiment [6] at the initial time but grows rapidly as the hot bubble shrinks. In our numerical simulations, $u \gg 1$. Eq. (11) takes the form (we drop tildes in what follows)

$$
\partial_{t} \psi=\Delta \psi+\partial_{x} \psi-\frac{x}{u} \psi-|\psi|^{2} \psi
$$

The amplitude $F$ of the current-carrying solution $\psi=$ $F \exp (i k y)$ satisfies

$$
\partial_{x}^{2} F+\partial_{x} F-\left(\frac{x}{u}+k^{2}\right) F-F^{3}=0 .
$$

To examine the transverse stability of stationary solution to Eq. (3) we put $\psi=(F+w) \exp (i k x)$ where the real and imaginary parts of the perturbation $w=a+i b$ are

$$
\left(\begin{array}{l}
a \\
b
\end{array}\right)=\left(\begin{array}{c}
A \\
i B
\end{array}\right) \exp (\lambda(q) t+i q y)
$$

where $q$ is the transverse undulations wavenumber and $\lambda$ is the corresponding growth rate, we obtain

$$
\begin{aligned}
& \Lambda A+2 \chi B=\partial_{x}^{2} A+\partial_{x} A-\frac{x}{u} A-3 F^{2} A \\
& \Lambda B+2 \chi A=\partial_{x}^{2} B+\partial_{x} B-\frac{x}{u} B-F^{2} B
\end{aligned}
$$

where $\chi=k q, \Lambda=\lambda+q^{2}$, and $x \rightarrow x+u k^{2}$.
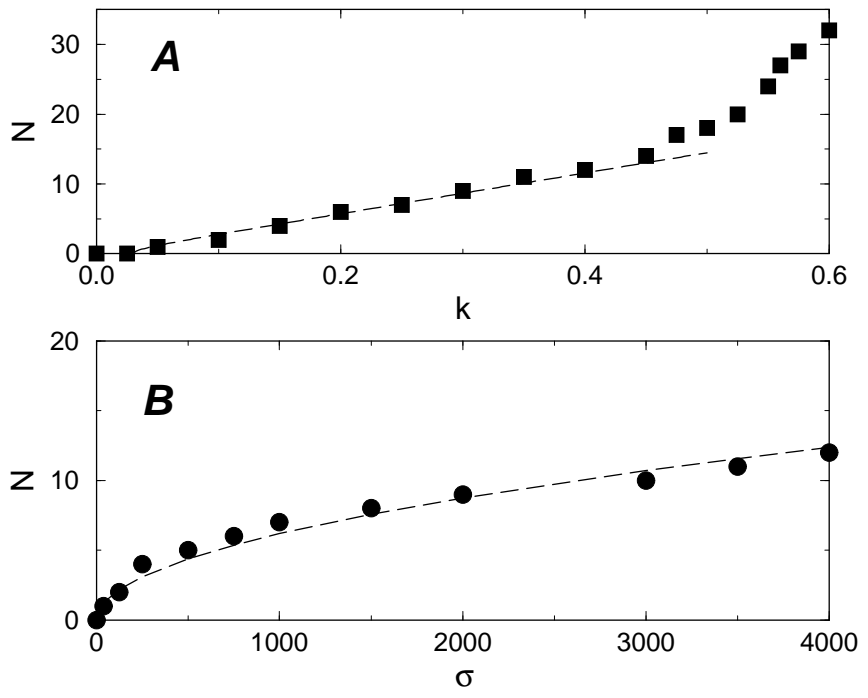

FIG. 4. (a) Number of vortex rings $N$ as function of $k$ for $E_{0}=50$ and $\sigma=5000$ and (b) $N$ vs $\sigma$ for $k=0.4$ and $E_{0}=50$. Dashed lines show fitting to prediction Eq. (14.).

The eigenvalue $\Lambda$ for $\chi \rightarrow 0$ can be found as an expansion in $\chi: \Lambda=\chi \Lambda_{1}+\chi^{2} \Lambda_{2}^{2}+\ldots$ and similarly for $A$ and $B$. In zeroth order in $\chi$ one has $A_{0}=0, B_{0}=F$. In the first order we derive $B_{1}=0$ and

$$
\partial_{x}^{2} A_{1}+\partial_{x} A_{1}-\frac{x}{u} A_{1}-3 F^{2} A_{1}=2 F .
$$

The solution $A_{1}=2 u \partial_{x} F$ is obtained by differentiating Eq. (3). In the second order to Eq. (5) one has

$$
\partial_{x}^{2} B_{2}+\partial_{x} B_{2}-\frac{x}{u} B_{2}-F^{2} B_{2}=4 u \partial_{x} F+\Lambda_{2} F .
$$

A zero mode of Eq. (7) is $F$. The adjoint function is $B^{+}=F \exp (x)$. Eq. (7) has a solution if the solvability condition with respect to the zero mode is fulfilled

$$
\int_{-\infty}^{\infty} d x F e^{x}\left(4 u \partial_{x} F+\Lambda_{2} F\right)=0
$$


After integration we obtain $\Lambda_{2}=2 u$. Returning to the original notations, we obtain the exact result

$$
\lambda=q^{2}\left(2 u k^{2}-1\right)+O\left(q^{4}\right)
$$

The instability occurs above the threshold value $k_{v}^{2}=$ $(2 u)^{-1}$ or $k_{v}^{2} \sim \alpha^{2 / 3} / u^{1 / 3} \sim \sigma^{-1} \log \left(t_{\max } / t\right)$ in the Ginzburg-Landau units. Since it is much smaller than the bulk critical value $k_{c}=1 / \sqrt{3}$, it can be exceeded for a very small superflow.

The eigenvalue $\Lambda$ vs $\chi$ and $u$ can be derived independently in the limit $u \gg 1$ assuming that $\Lambda \sim \chi \sim 1 / u \ll$ 1. Substituting $x=\bar{x}-u \gamma$, where $\gamma$ determines the position of the interface, we treat the terms containing $\Lambda, \chi$ and $\bar{x} / u$ as perturbations for $\epsilon=1 / u \rightarrow 0$. For $\epsilon=0$ and $\gamma>1 / 4$ Eqs. (3) possess a front solution. This solution should be matched with its asymptotics for $\bar{x}>0$, and this match fixes the value of $\gamma$. As it was shown in Ref. [7], for $u \rightarrow \infty$ the matching is possible for $\gamma \rightarrow 1 / 4$.

For $\epsilon=0$ Eqs. (5) have 2 zero modes: $(A, B)=\left(F_{x}, 0\right)$ and $(A, B)=(0, F)$. The solvability conditions result in the characteristic equation for $\Lambda$ :

$$
\Lambda^{2}+\frac{1}{u} c_{1} \Lambda-4 c_{2} \chi^{2}+\frac{d}{u^{2}}=0,
$$

where the coefficients $c_{1,2}, d$ are given in the forms of integrals of $F$ with the corresponding zero modes in the interval $-\infty<\bar{x}<x_{0}$. The constant $x_{0}$ is determined from the condition $d=0$ because for $\chi=0$ there is always an exact solution to Eq. (5) with $\Lambda=0$. Substitution of the solutions for $\gamma \rightarrow 1 / 4$ yields $c_{1} \rightarrow 2, c_{2} \rightarrow 1$ and the largest growthrate of transverse perturbations

$$
\lambda=\sqrt{1 / u^{2}+4 k^{2} q^{2}}-1 / u-q^{2} .
$$

Numerical solution of Eqs. (5) demonstrates an excellent agreement with the theoretical expression Eq. (11).

Now we apply the above results to estimate the number of nucleated vortices. The evolution of perturbations near the interface is given by the integral

$$
w \sim \int d q \exp [\lambda(q) t+i q y] .
$$

In the case of thermal quench, the normal/superfluid front velocity $u \rightarrow \infty$ as time increases, therefore, the limit of large $u$ applies. For $u \rightarrow \infty$ one has $\lambda=2|k q|-q^{2}$. The maximum growth rate is achieved at $q=k$ and is simply $k^{2}$. Taking into account that it is the thermal noise which provides initial perturbations for the interface instability, one derives from Eqs. (11, 12) $\langle|w|\rangle \sim$ $\sqrt{T_{f}} \exp \left[k^{2} t+i k y\right]$. The number of vortices is estimated as $N=r_{0} k$, where $r_{0}$ is the radius of the front where the perturbations $w$ become of the order of one. The time interval $t_{0}$ corresponding to $\langle|w|\rangle=1$ is $t_{0} \sim k^{-2} \log \left(T_{f}^{-1}\right)$. Vortices have no time to grow if $t_{0} \rightarrow t_{\max }$. For $r_{0}$ one then finds:

$$
r_{0}^{2}=(3 / 2) \sigma\left(t_{\max }-t_{0}\right) \log \left(t_{\max } /\left(t_{\max }-t_{0}\right)\right) .
$$

The number of vortices with logarithmic accuracy is

$$
N \sim k r_{0} \sim \sqrt{\sigma} E_{0}^{1 / 3} \sqrt{\left(v_{s} / v_{c}\right)^{2}-\beta^{2} \log \left(T_{f}^{-1}\right) / E_{0}^{2 / 3}}
$$

where $\beta=$ const, while $v_{s}$ and $v_{c}$ are the imposed and critical GL superflow velocity, respectively. This estimate is in agreement with the results of simulations, see Fig. 4. Eq. (14) exhibits a slow logarithmic dependence of the number of vortices at the interface on the level of fluctuations and agrees with the results presented in Fig. 3 [9]. For $\sigma \sim 10^{3}, E_{0} \sim 10^{2}-10^{3}$ which is close to the experimental values of the parameters. Our analysis results in about 10 surviving vortices per heating event. It is consistent with Ref. [6] where as many as 6 vortices per neutron were detected.

In conclusion, we have found that the rapid normalsuperfluid transition in the presence of superflow is dominated by a transverse instability of the normal/superfluid interface propagating from the bulk into the normal region. This instability produces primary vortex loops which then separate from the interface. Simultaneously, a large number of vortex/antivortex pairs are created by fluctuations in the bulk of the supercooled region formed after the collapse of the hot bubble. The primary vortex loops screen out the superflow and cause annihilation of the vortex/antivortex pairs in the bulk. The number of surviving vortices is determined by superflow-dependent optimum wavevector of the interface instability.

We are grateful to V. Eltsov, M. Krusius, G. Volovik and W. Zurek for stimulating discussions. This research is supported by US DOE, grant W-31-109-ENG-38, and by NSF, STCS \#DMR91-20000.

[1] T.W.B. Kibble, J. Phys. A: Math Gen 9, 1387 (1976)

[2] W. H. Zurek, Nature 317, 505 (1985)

[3] N.D. Antunes, L.M.A. Bettencourt, and W.H. Zurek, Phys. Rev. Lett. 82, 2824, (1999)

[4] T.W.B. Kibble and G.E. Volovik, JETP Lett. 65, 102 (1997)

55] V.B. Eltsov, M. Krusius, and G.E. Volovik, condmat/9809125, to be published.

[6] V.M.H. Ruutu et al, Nature 382, 334 (1996); V.M.H. Ruutu et al, Phys. Rev. Lett. 80, 1465 (1998).

[7] N.B. Kopnin and E.V. Thuneberg, submitted to Phys. Rev. Lett. (1999)

[8] Even for $T_{f}=0$ there is a small level of fluctuation due to roundoff effects in numerical integration. For single precision it may generate noise with the strength $T_{f} \sim 10^{-6}$.

[9] Eq. (14) describes only effect of interface instability where as Fig. 3 displays outcome of whole nucleation process both in the bulk and the interface. 